\title{
General Psychiatry Psychological impact of the COVID-19 pandemic on healthcare workers: a cross-sectional study in China
}

\author{
Jianyu Que (1) , ${ }^{1}$ Le Shi, ${ }^{1}$ Jiahui Deng, ${ }^{1}$ Jiajia Liu, ${ }^{1,2}$ Li Zhang, ${ }^{1,3}$ Suying Wu, ${ }^{4}$ \\ Yimiao Gong, ${ }^{1,5}$ Weizhen Huang, ${ }^{1}$ Kai Yuan, ${ }^{1}$ Wei Yan, ${ }^{1}$ Yankun Sun, ${ }^{1}$ \\ Maosheng Ran, ${ }^{6}$ Yanping Bao, ${ }^{2}$ Lin $\mathrm{Lu}^{1,2,5}$
}

To cite: Que J, Shi L, Deng J, et al. Psychological impact of the COVID-19 pandemic on healthcare workers: a cross-sectional study in China. General Psychiatry 2020;33:e100259. doi:10.1136/ gpsych-2020-100259

JQ and LS contributed equally.

Received 26 April 2020 Revised 16 May 2020 Accepted 23 May 2020
Check for updates

(c) Author(s) (or their employer(s)) 2020. Re-use permitted under CC BY-NC. No commercial re-use. See rights and permissions. Published by BMJ.

For numbered affiliations see end of article.

Correspondence to Professor Lin Lu; linlu@bjmu.edu.cn

Professor Yanping Bao; baoyp@bjmu.edu.cn

\section{ABSTRACT}

Background Healthcare workers fighting against the coronavirus disease 2019 (COVID-19) pandemic are under tremendous pressure, which puts them at an increased risk of developing psychological problems. Aims This study aimed to investigate the prevalence of psychological problems in different healthcare workers (ie, physicians, medical residents, nurses, technicians and public health professionals) during the COVID-19 pandemic in China and explore factors that are associated with the onset of psychological problems in this population during this public health crisis.

Methods A cross-sectional, web-based survey was conducted in February 2020 among healthcare workers during the COVID-19 pandemic. Psychological problems were assessed using the Generalized Anxiety Disorder Scale, Patient Health Questionnaire and Insomnia Severity Index. Logistic regression analyses were used to explore the factors that were associated with psychological problems.

Results The prevalence of symptoms of anxiety, depression, insomnia and the overall psychological problems in healthcare workers during the COVID-19 pandemic in China was $46.04 \%, 44.37 \%, 28.75 \%$ and $56.59 \%$, respectively. The prevalence of the overall psychological problems in physicians, medical residents, nurses, technicians and public health professionals was $60.35 \%, 50.82 \%, 62.02 \%, 57.54 \%$ and $62.40 \%$, respectively. Compared with healthcare workers who did not participate in front-line work, front-line healthcare workers had a higher risk of anxiety, insomnia and overall psychological problems. In addition, attention to negative or neutral information about the pandemic, receiving negative feedback from families and friends who joined front-line work, and unwillingness to join front-line work if given a free choice were three major factors for these psychological problems.

Conclusions Psychological problems are pervasive among healthcare workers during the COVID-19 pandemic. Receiving negative information and participating in front-line work appear to be important risk factors for psychological problems. The psychological health of different healthcare workers should be protected during the COVID-19 pandemic with timely interventions and proper information feedback.

\section{INTRODUCTION}

The dramatic spread of coronavirus 2019 (COVID-19) pandemic worldwide has caused a tremendous public health crisis. ${ }^{12}$ Across the globe, a greater number of fatalities by COVID-19 have been reported than those by the previous severe acute respiratory syndrome (SARS) and Middle East respiratory syndrome combined, even though COVID-19 has a relatively low death rate. ${ }^{3}$ However, to date, no effective treatment has been developed to cure people with COVID19. ${ }^{4}$ With increasing number of confirmed cases and death counts due to COVID-19, this overwhelming global pandemic poses a great challenge to the local healthcare systems. As the number of patients with COVID-19 grows, increasingly more health resources, including personnel, beds and facilities, are at maximum capacity. With limited resources, people will be under greater pressure and experience greater distress, especially healthcare workers. ${ }^{56}$

To control the transmission of the offending virus, severe acute respiratory syndrome coronavirus 2, and treat people with COVID-19, many medical measures have been implemented in different countries. By 8 April 2020, according to the WHO, 22073 confirmed cases of COVID-19 have been reported among healthcare workers in 52 countries. ${ }^{1}$ More than 42000 healthcare workers in China, including physicians, nurses, technicians and public health professionals, from other provinces went to Hubei Province to provide assistance. ${ }^{7}$ In addition to supporting Hubei, different healthcare workers including physicians, nurses, technicians and public health staff in other provinces were divided into several groups to establish fever clinics, manage individuals with COVID-19 and control the infection among medical staff. ${ }^{8}$ To ensure the quality of 
medical services, several researchers have appealed to the authorities to take the necessary steps for strengthening mental health and wellness for healthcare workers. ${ }^{9} 10$

Compared with the general population, healthcare workers are facing tremendous pressure from COVID-19, especially those who might be in contact with suspected or confirmed cases, due to the high risk of infection, inadequate protection, loss of control, lack of experience in managing the disease, overwork, negative feedback from patients, perceived stigma, significant lifestyle changes, quarantine and less family support. ${ }^{11-13}$ These factors increase the incidence of psychological problems among healthcare workers, such as fear, anxiety, depression and insomnia, which can negatively affect work efficiency and long-term well-being. ${ }^{15}$ During the SARS epidemic, $29 \%-35 \%$ of hospital workers suffered from a high degree of emotional distress. ${ }^{13}$ Even several years later, $10 \%$ of healthcare workers still reported symptoms of post-traumatic stress. ${ }^{16}$ Individuals who experienced quarantine or worked in wards for patients with infection were two to three times more likely to have post-traumatic stress symptoms. ${ }^{16}$ Although a few studies have investigated the prevalence of psychological problems among healthcare workers during the COVID-19 pandemic, ${ }^{17} 18$ no study has investigated the distribution of psychological problems among different groups of healthcare workers.

A more comprehensive understanding of psychological burden among different groups of healthcare workers during this period is crucial for providing psychological support, improving mental health support services and strengthening mental healthcare worldwide. ${ }^{19}$ This crosssectional study investigated the prevalence of psychological problems in different healthcare workers during the COVID-19 pandemic in China and explored the demographics and COVID-19-related and work-related factors that are associated with various psychological problems.

\section{MATERIALS AND METHODS \\ Study design}

An anonymous cross-sectional study was performed using the social media platform-based (WeChat) survey program Questionnaire Star in February 2020 in China. Healthcare professionals, including physicians, medical residents, nurses, technicians and public health professionals, were invited to voluntarily participate in the selfadministered online survey. The detailed flowchart of this study is shown in figure 1. At the beginning of the questionnaire, we informed participants that they would be signing the consent by default if they completed the survey.

\section{Study sample}

Healthcare workers from different regions throughout China were recruited based on social networks of investigators and research teams, in order to reach a large number of subjects. The participants were contacted via a designated link, which was disseminated through the
Medical professionals from different regions of China were invited to participate in this online survey

(1)

Figure 1 Flow chart of subjects' enrollment.

primary means of communication and social networks of each participant. Those who are not medical professionals, are non-users of WeChat or did not complete the assessment were not involved in this survey. Medical students were excluded in this analysis because most of them do not enter the stage of clinical practice. We analysed the data collected from 16 February to 23 February 2020, at the early stage of the COVID-19 pandemic, until a projected sample size was reached based on estimates of the prevalence of psychological problems during the SARS outbreak. ${ }^{13}$

\section{Measurements}

We developed a questionnaire to collect data on demographic characteristics and psychological status of subjects and information about the COVID-19 pandemic. The duration of the questionnaire was approximately $10 \mathrm{~min}$. Demographic data, including age, gender, race, years of education, annual household income, geographical region and occupation, were obtained. We assessed regular exercise and hazardous drinking and smoking by asking the respondents the following questions: 'Did you engage in physical exercise for $150 \mathrm{~min}$ or more per week?'20 'Did you drink regularly more than 14 units of alcohol (equivalent to six pints of beer or six glasses of wine) per week? ${ }^{21}$ and 'Did you smoke 10 or more cigarettes per day for one or more years?" 22 The respondents responded 'yes' or 'no'.

The Chinese version of the Seven-Item Generalized Anxiety Disorder Scale (GAD-7) was used to measure symptoms of anxiety during the past 2 weeks. ${ }^{23}$ Cutoff points of 5, 10 and 15 were classified as mild, moderate and severe anxiety, respectively. ${ }^{24}$ The diagnostic threshold of the GAD-7 was previously reported to be $10 .{ }^{25}$ We identified depressive symptoms during the past 2 weeks using the Chinese version of the NineItem Patient Health Questionnaire (PHQ-9), which has shown high consistency with a diagnosis of major depression based on structured interviews. ${ }^{26}{ }^{27}$ Cut-off 
points of 5, 10 and 15 were classified as mild, moderate and severe depression, respectively. A PHQ-9 score $\geq 10$ was previously reported to have a sensitivity of $88 \%$ and specificity of $88 \%$ for major depression. ${ }^{28}$ Symptoms of insomnia were measured using the Chinese version of the Insomnia Severity Index (ISI) ${ }^{29}$ Cut-off points of 8,15 and 22 were classified as subthreshold, moderate and severe insomnia, respectively. ${ }^{30}$ An ISI score $\geq 15$ was defined as insomnia, based on a diagnostic utility study. ${ }^{31}$ In this study, we also assessed the overall psychological problems. The overall mild psychological problems were defined as any symptom of mild anxiety, depressive or insomnia, and the overall moderate/ severe psychological problems were defined as any symptom of moderate/severe anxiety, depressive or insomnia.

We also collected a series of information about exposure to COVID-19. We assessed the influence of the COVID-19 pandemic by asking four questions. First, we asked 'Do you pay attention to information about the COVID-19 pandemic?' Responses were dichotomised as 'never/seldom' and 'often/always'. Second, we asked 'What kind of information about the pandemic are you concerned about?' The response options were 'positive', 'neutral' and 'negative'. Third, we asked 'Did you receive feedback from your families or friends who joined front-line work?' The response options were 'no', 'yes, he/she is satisfied with front-line work' and 'yes, he/she is unsatisfied with front-line work'. Fourth, we asked 'Is anyone close to you suspected of being infected or confirmed to be infected?' The response options were 'yes' or 'no'. Front-line work was defined as jobs in which one may be in contact with suspected or confirmed cases. The questions 'Did you participate in front-line work?' and 'Were you suspected of being infected or confirmed to be infected during front-line work?' were used to assess the effects of participation in front-line work on psychological problems. We also explored the association between psychological problems and the willingness to join front-line work by asking 'If given a free choice, did you want to join frontline work?' The response options were 'willing', 'uncertain' and 'unwilling'.

\section{Data analysis}

We used descriptive statistical analysis to characterise the samples of healthcare workers. The prevalence of symptoms of anxiety, depression, insomnia and the overall psychological problems was reported. According to previous studies, a GAD-7 score $\geq 10$ indicates anxiety. ${ }^{25}$ A PHQ-9 score $\geq 10$ indicates depression. ${ }^{28}$ An ISI score $\geq 15$ indicates clinical insomnia. ${ }^{31}$ GAD-7, PHQ-9 and ISI scores were treated as dichotomous variables in the univariate analysis. We performed unadjusted logistic regression analyses to investigate factors that are associated with anxiety, depression, insomnia and the overall psychological problems. Variables were included in the multiple backward logistic regression analysis if they had $\mathrm{p}$ values $<0.05$ in the unadjusted analysis. The results are reported as adjusted ORs with 95\% CIs. All of the tests were two-sided. $\mathrm{P}<0.05$ was considered statistically significant. SPSS V.20 software was used for statistical analyses.

\section{RESULTS}

\section{Demographic characteristics}

A total of 2285 healthcare workers from 28 province-level regions in China completed the questionnaire, of whom $707(30.94 \%)$ were men and 1578 (69.06\%) were women, with an average age of 31.06 years ( $\mathrm{SD}=6.99$ years). The participants included medical residents $(913 ; 39.96 \%)$, physicians $(860 ; 37.64 \%)$, nurses $(208 ; 9.10 \%)$, technicians $(179 ; 7.83 \%)$ and public health practitioners $(125$; $5.47 \%)$. The respondents' years of education varied from 11 years to 30 years, with an average of 18.25 years $(\mathrm{SD}=2.34$ years $)$. Among the respondents, $43.46 \%$ were from eastern China, $33.74 \%$ were from western China, $15.40 \%$ were from central China and 5.82\% were from northeastern China. The majority of the respondents worked in tertiary hospitals (table 1).

\section{Prevalence of psychological problems in healthcare workers}

Table 2 presents the prevalence of anxiety, depression, insomnia and the overall psychological problems in healthcare workers. For anxiety, $46.04 \%$ of the healthcare workers had GAD-7 scores $\geq 5$, including $34.44 \%$ with mild anxiety and $11.60 \%$ with moderate/severe anxiety. The highest prevalence of anxiety symptoms was observed in nurses $(51.44 \%)$. The lowest prevalence of anxiety symptoms was observed in medical residents $(38.99 \%)$. For depression, $44.37 \%$ of the healthcare workers had PHQ-9 scores $\geq 5$, including $31.55 \%$ with mild depression and $12.82 \%$ with moderate/severe depression. The highest prevalence of depressive symptoms was observed in public health professionals $(48.80 \%)$. The lowest prevalence of depressive symptoms was observed in medical residents $(40.53 \%)$. For insomnia, $28.75 \%$ of the healthcare workers had ISI scores $\geq 8$, including $21.97 \%$ with subthreshold insomnia and $6.78 \%$ with moderate/severe insomnia. The highest prevalence of insomnia symptoms was observed in nurses $(33.17 \%)$. The lowest prevalence of insomnia symptoms was observed in medical residents $(24.53 \%)$. For the overall psychological problems, $56.59 \%$ of the healthcare workers had symptoms of anxiety, depression or insomnia, including $38.47 \%$ with mild symptoms and $18.12 \%$ with moderate/severe symptoms. The highest prevalence of psychological problems was observed in public health professionals $(62.40 \%)$. The lowest prevalence of insomnia symptoms was observed in medical residents $(50.82 \%)$.

\section{Factors associated with psychological problems}

We performed binary logistic regression analyses to identify demographic and relevant contextual factors that are associated with psychological problems (table 3). In the unadjusted logistic regression analysis, several factors 


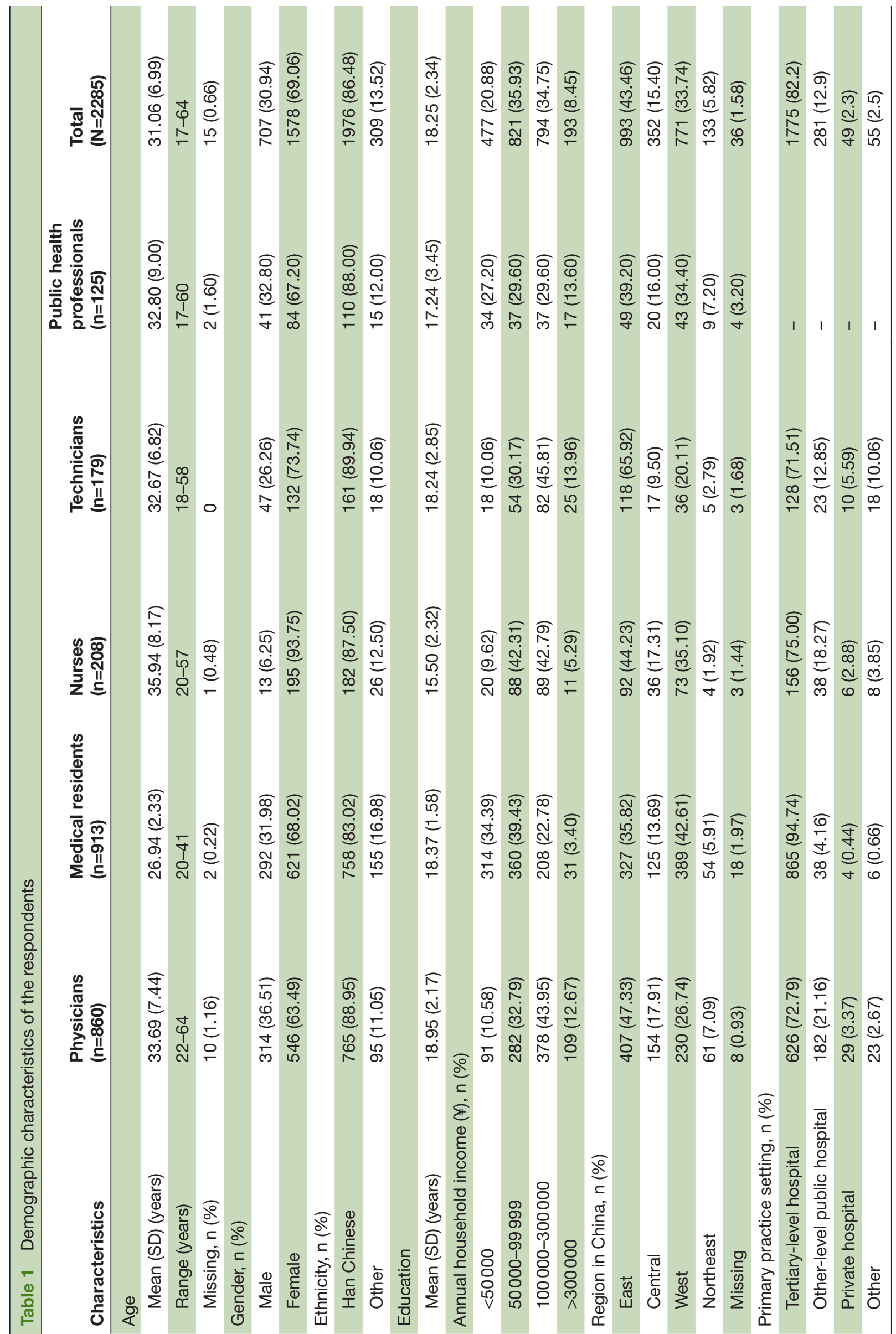




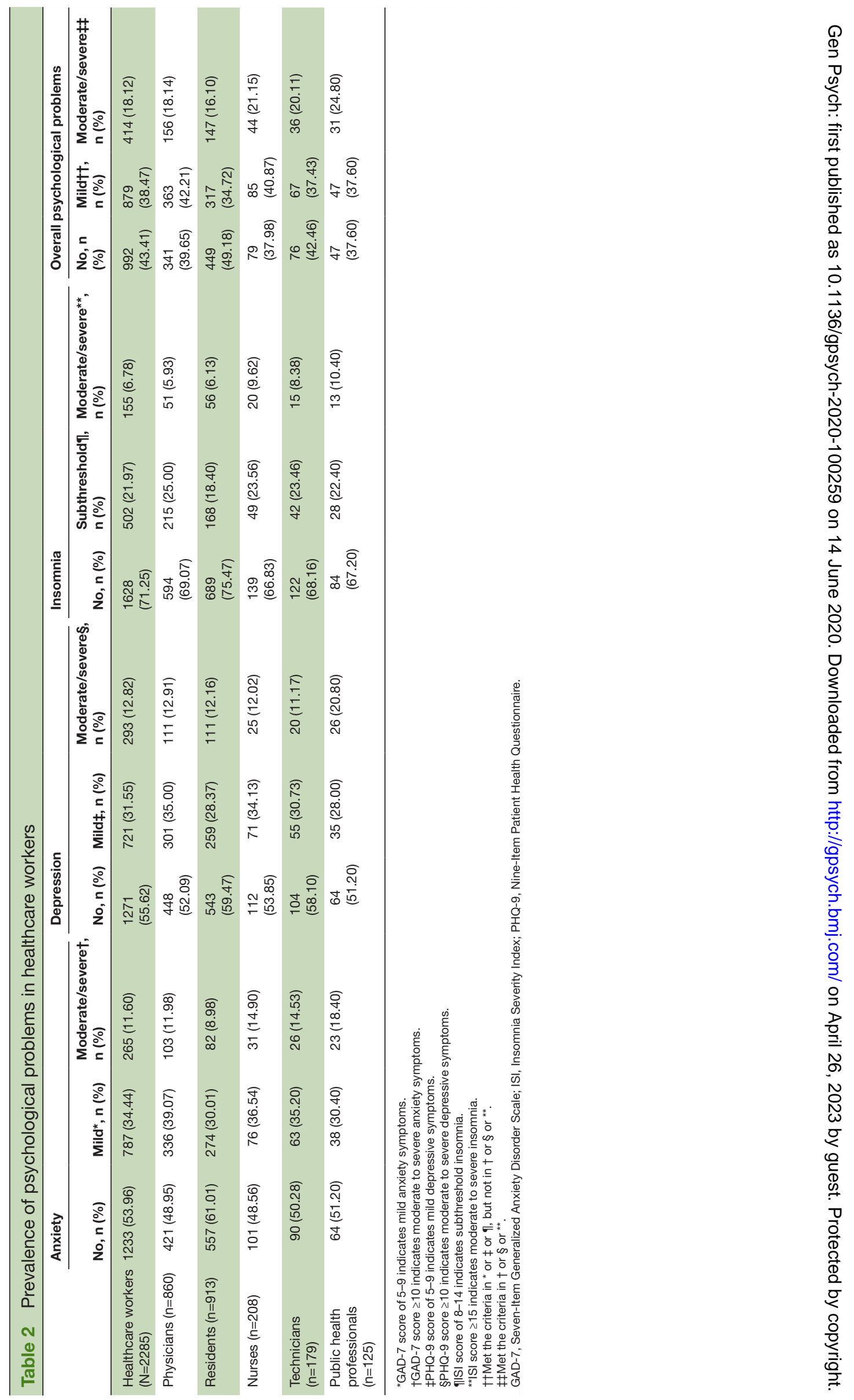




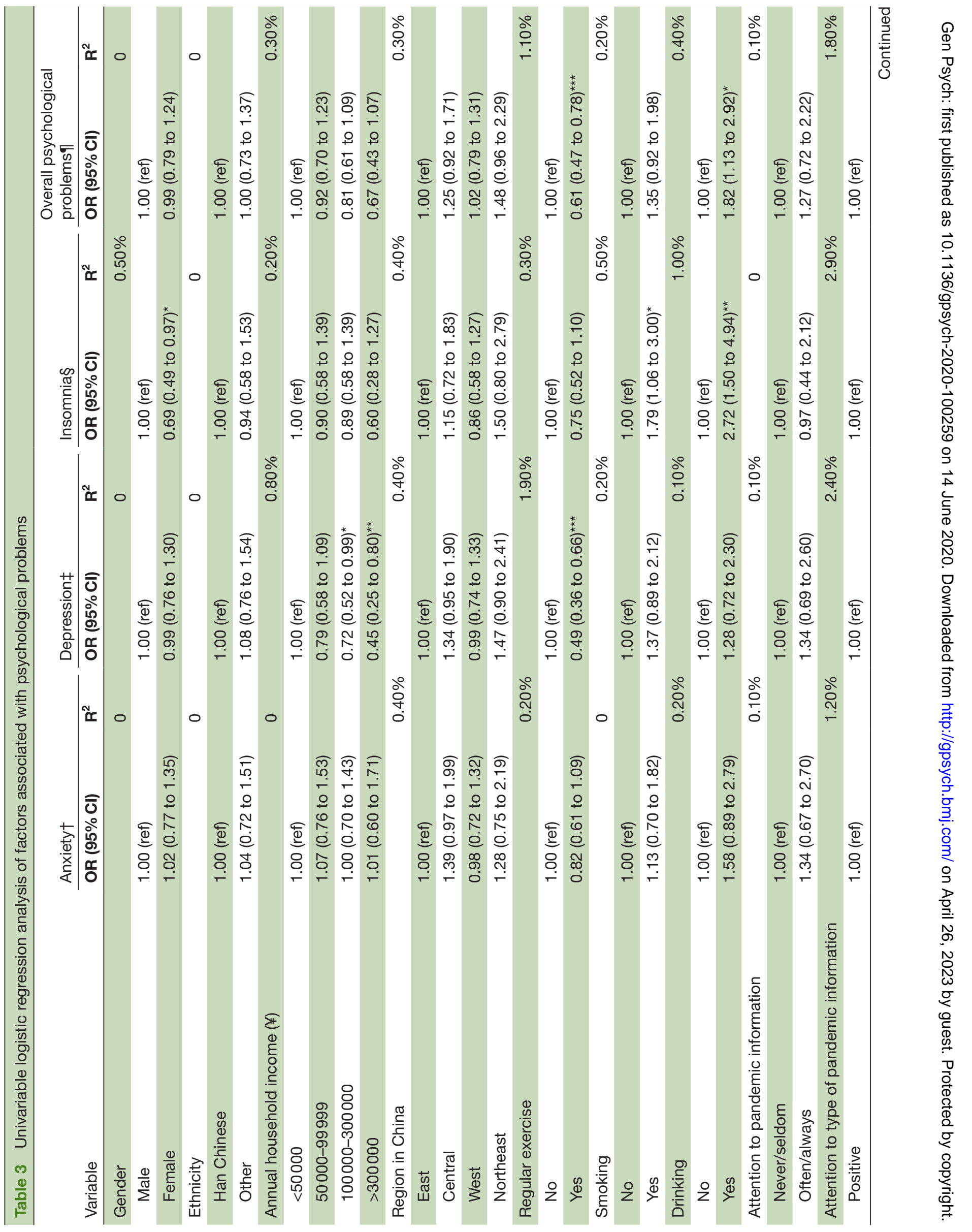




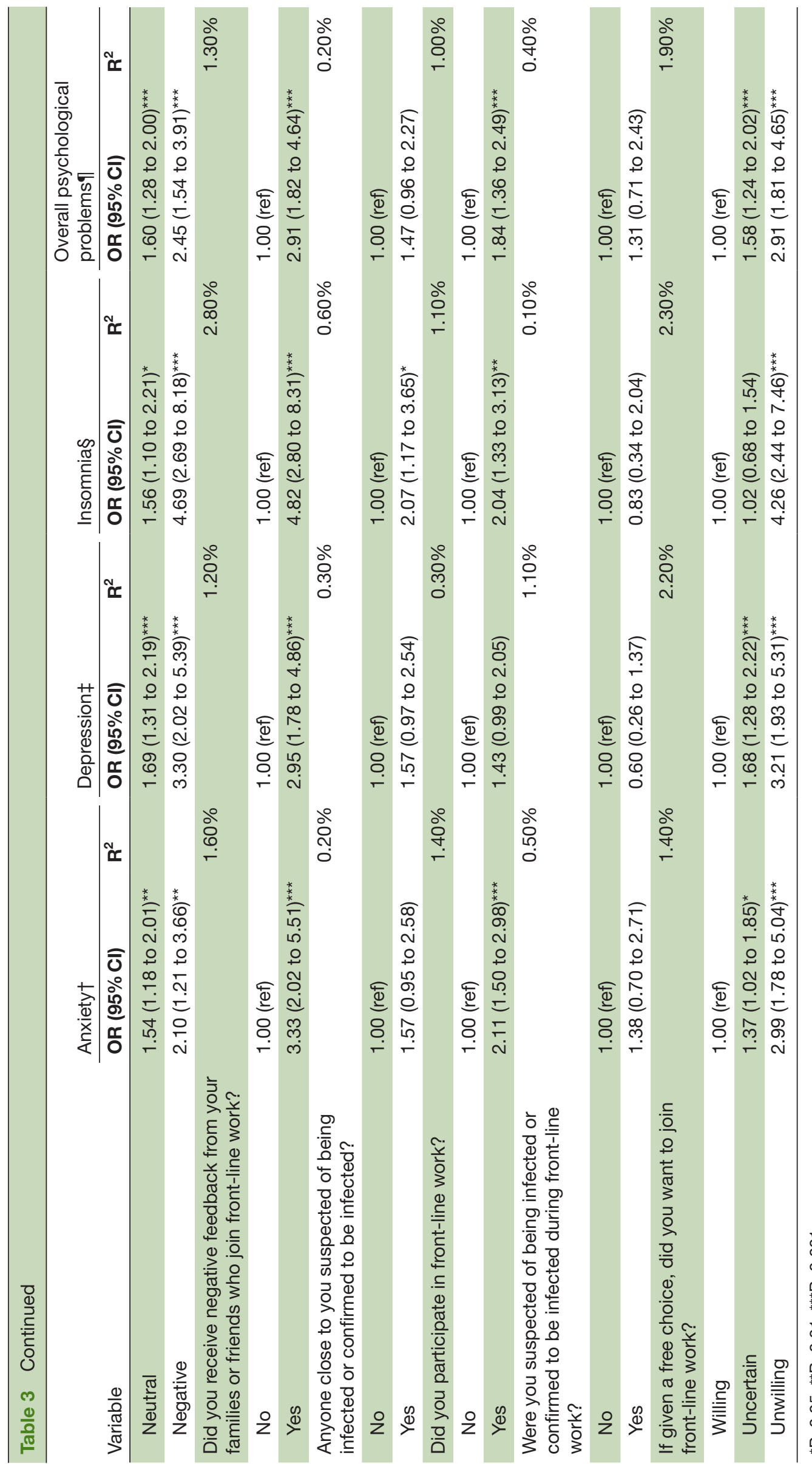

ه

0

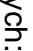

$\vec{F}$

음

ब

@

$\vec{\circ}$

$\overrightarrow{\vec{\omega}}$

응

웅

กิ

음 
were independently associated with anxiety (GAD-7 score $\geq 10$ ), depression (PHQ-9 score $\geq 10$ ), insomnia (ISI score $\geq 15$ ) and the overall moderate/severe psychological problems.

In the multiple logistic analyses, factors that were independently associated with a higher risk of anxiety included attention to neutral information about the pandemic $(\mathrm{OR}=1.38,95 \%$ CI 1.04 to 1.83$)$, receiving negative feedback from families or friends who joined front-line work $(\mathrm{OR}=2.53,95 \%$ CI 1.50 to 4.26), joining front-line work $(\mathrm{OR}=2.12,95 \% \mathrm{CI} 1.49$ to 3.02$)$ and unwilling to join front-line work if given a free choice $(\mathrm{OR}=2.57,95 \% \mathrm{CI}$ 1.50 to 4.43$)$.

High annual household income ( $¥ 100000-¥ 300000$ vs $<¥ 50000, \mathrm{OR}=0.64,95 \%$ CI 0.46 to 0.89 ; $>¥ 300000$ vs $<¥ 50000, \mathrm{OR}=0.34,95 \%$ CI 0.19 to 0.63 ) and regular exercise (OR=0.51, 95\% CI 0.37 to 0.70 ) were independently associated with a lower risk of depression. Factors that were independently associated with a higher risk of depression included attention to negative information about the pandemic (OR=2.49, 95\% CI 1.48 to 4.18$)$, attention to neutral information about the pandemic ( $\mathrm{OR}=1.50,95 \%$ CI 1.14 to 1.97$)$, receiving negative feedback from families or friends who joined front-line work ( $\mathrm{OR}=2.96$, 95\% CI 1.73 to 5.06), unwilling to join frontline work if given a free choice $(\mathrm{OR}=2.38,95 \%$ CI 1.39 to $4.06)$ and uncertain about joining front-line work if given a free choice $(\mathrm{OR}=1.47,95 \%$ CI 1.10 to 1.97$)$.

Factors that were independently associated with a higher risk of insomnia included drinking $(\mathrm{OR}=2.43$, $95 \%$ CI 1.29 to 4.55 ), attention to negative information about the pandemic (OR=3.34, 95\% CI 1.84 to 6.06), receiving negative feedback from families or friends who joined front-line work (OR=3.47, 95\% CI 1.95 to 6.17$)$, joining front-line work (OR=1.90, 95\% CI 1.21 to 2.97) and unwilling to join front-line work if given a free choice $(\mathrm{OR}=3.39,95 \% \mathrm{CI} 1.86$ to 6.17$)$.

Regular exercise $(\mathrm{OR}=0.62,95 \%$ CI 0.48 to 0.80$)$ was a protective factor against the overall psychological problems. However, drinking ( $\mathrm{OR}=2.06,95 \%$ CI 1.26 to 3.36), attention to neutral pandemic information $(\mathrm{OR}=1.41$, $95 \% \mathrm{CI} 1.21$ to 1.78$)$, attention to negative pandemic information ( $\mathrm{OR}=1.81,95 \% \mathrm{CI} 1.11$ to 2.94$)$, receiving negative feedback from families or friends who joined front-line work ( $\mathrm{OR}=2.38,95 \%$ CI 1.46 to 3.87), joining front-line work (OR=1.85, 95\% CI 1.35 to 2.54), unwilling to join front-line work if given a free choice $(\mathrm{OR}=2.36$, $95 \%$ CI 1.44 to 3.85 ) and uncertain about joining frontline work if given a free choice $(\mathrm{OR}=1.49,95 \% \mathrm{CI} 1.15$ to 1.93) were independently associated with increased risk of overall psychological problems (table 4).

\section{DISCUSSION}

\section{Main findings}

The present results show that self-reported psychological problems are prevalent in healthcare workers during the COVID-19 pandemic. Moreover, different kinds of healthcare workers exhibited a distinct prevalence of anxiety, depression, insomnia and overall psychological problems. We further identified the possible risk factors associated with psychological problems, including participation in front-line work, attention to neutral or negative information about the pandemic, receiving negative feedback from people who worked on the front-line, and uncertainty or unwillingness to join front-line work and so on. The findings help provide information for psychological interventions among healthcare workers in other countries and religions.

The high prevalence of psychological problems that was found in this study is consistent with recent findings from two other Chinese research studies with relatively small samples. ${ }^{1732}$ The prevalence of self-reported symptoms of anxiety, depression and insomnia in these two previous surveys was $44.7 \%, 50.7 \%$ and $36.1 \%{ }^{32}$ and $44.6 \%, 50.4 \%$ and $34.0 \%,{ }^{17}$ respectively. Another study confirmed the severe mental health conditions in healthcare workers and indicated that medical health workers reported more symptoms compared with non-medical health workers. ${ }^{18}$ In addition, compared with the general population (eg, $34.43 \%$ of the general population experienced psychological distress), ${ }^{33}$ healthcare workers have a much higher risk of psychological problems (eg, anxiety, depression and insomnia) during the epidemic. ${ }^{15} 3435$ This may be related to the higher risk of infection on account of being exposed to patients with COVID-19 and tedious work involved in caring for them and reminds us of the importance of providing psychological support to healthcare workers during a pandemic.

Moreover, this study analysed the prevalence of psychological problems in different healthcare workers, including nurses, physicians, medical residents, technicians and public health professionals. Approximately one-third to half of the nurses reported symptoms of anxiety, depression and insomnia. Nurses, mostly women who are more susceptible to mental problems, may have a higher workload and greater risk of direct exposure to patients with COVID-19. ${ }^{36}$ Moreover, owing to the contagious nature of COVID-19, as a preventive measure, nurses may be separated from their family members to reduce the risk of transmission, and research has shown that worrying about family members may be one of the main sources of stress in nurses, indicating the critical role of community support for nurses' mental health. ${ }^{37}$ Similar to nurses, physicians are also under great stress, and nearly half of the physicians reported anxiety and depression. However, majority of the physicians are male and may have a higher acceptance of risk than nurses and have better coping skills. ${ }^{38}$ In this study, we also found a high prevalence of psychological problems in public health professionals and technicians. However, few studies have investigated their mental health during a pandemic, and further research is warranted to provide more evidence. During the COVID-19 pandemic, many medical residents do not directly participate in the care 


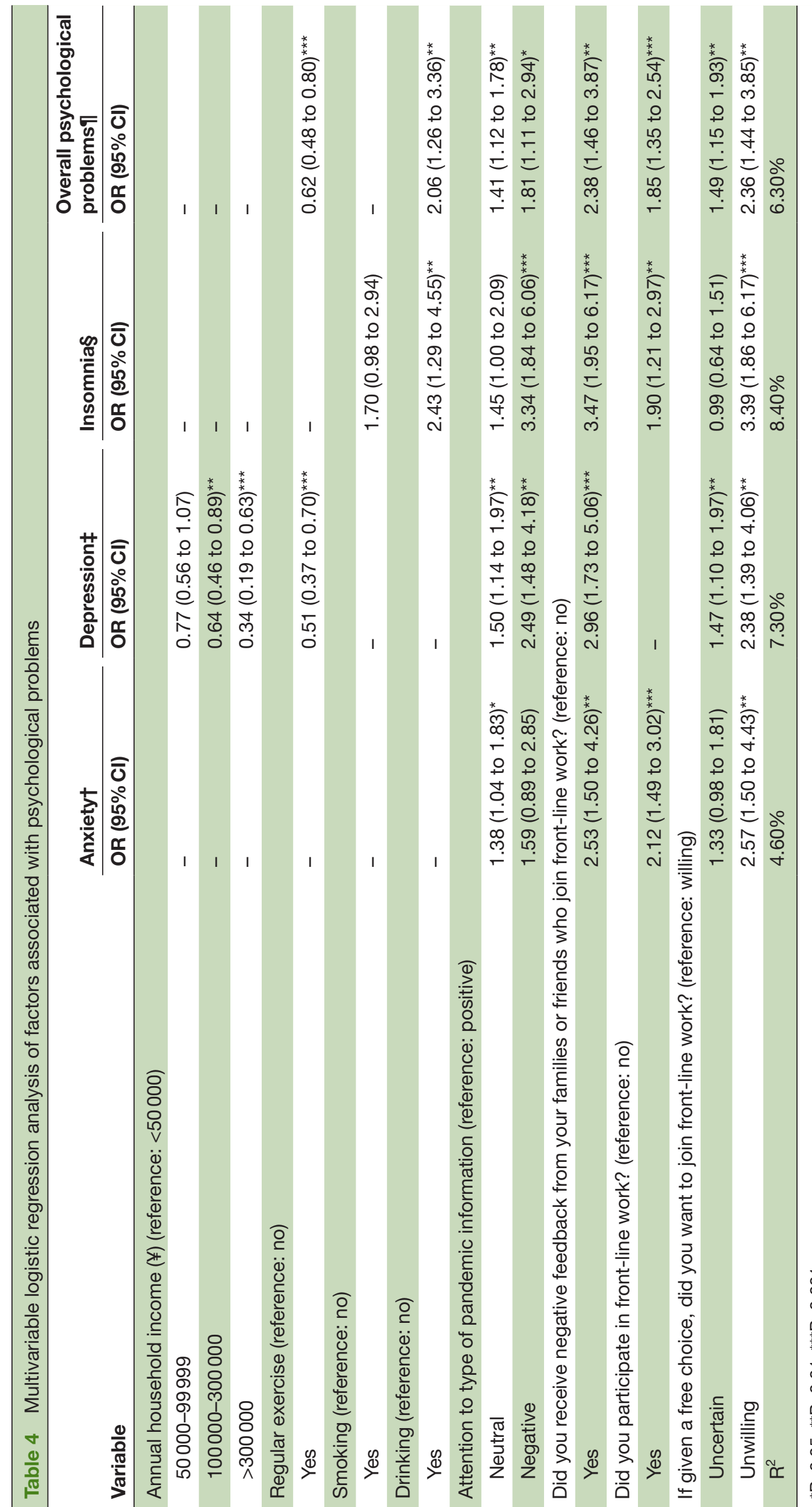

๑

0

’

$\overrightarrow{\bar{\sigma}}$

흠

$\widehat{\nabla}$

$\overrightarrow{\vec{\omega}}$

응

๙ุำ

No

호 
of patients with COVID-19 (eg, many medical residents may only be on stand-by at home during the COVID-19 pandemic), and thus, they reported less anxiety, depression, insomnia and overall psychological problems. ${ }^{40}$ These findings indicate that based on the sociodemographic, occupational and institutional disparities, different psychological interventions should be delivered to healthcare workers.

One interesting finding of this study was that paying attention to negative or neutral information (rather than positive information) about the COVID-19 pandemic and receiving negative feedback from families and friends who joined front-line work were associated with a higher risk of psychological problems. Exposure to a preponderance of negative information about a disaster and misinformation from the media and surrounding people can potentially damage mental health. ${ }^{41}{ }^{42}$ However, although individuals with psychological disorders might tend to focus on negative information about the COVID-19 pandemic, such information can also have a negative impact on their mental health, which may lead to a vicious circle. In addition, engaging in regular exercise might serve as a protective factor against depression and overall psychological problems, and a higher household income was also found to be protective against depression, which is consistent with previous studies. ${ }^{43}{ }^{44}$ We also found that hazardous drinking was independently associated with insomnia and overall psychological problems. ${ }^{45} 46$ These results demonstrated the importance of information control and a healthy lifestyle (eg, regular exercise and no hazardous drinking) in reducing psychological problems in healthcare workers.

Another intriguing finding in this study was that healthcare workers' unwillingness to join front-line work if given a free choice was independently associated with psychological problems, which is consistent with findings that were reported during the SARS epidemic. ${ }^{47}$ The major reason for unwillingness to work on the front-line is lack of confidence in infection control knowledge and skills. Individuals who perceive that they have a limited capacity in such knowledge and skills are more likely to experience psychological problems. ${ }^{14048}$ Healthcare workers' knowledge and skills need to be improved so that they are well equipped to cope with infectious disease, and steps should be taken to provide necessary support to reduce the risk of infection and improve the work environment. ${ }^{49}$

\section{Limitations}

This study has several limitations. This was a crosssectional online survey and the sample is not necessarily a well representation. The causal relationships should be interpreted with caution. Although a relatively large number of healthcare workers participated in this study, the limited number of participants in the northeast areas of China might have caused the findings to be underpowered. More studies are needed to explore the longitudinal trajectories of anxiety, depression and insomnia symptoms in healthcare workers during the COVID-19 pandemic in China. Moreover, the number of nurses, technicians and public health professionals who participated in this survey was limited, which may limit the generalisability of findings. The results were based on self-reported questionnaires that investigated psychological problems, which might be different from clinical diagnostic interviews.

\section{Implications}

The results of this study show the burden of psychological problems among different healthcare workers during the COVID-19 pandemic. The findings suggest that a greater risk of psychological problems may be associated with receiving negative information about the pandemic. Participation in front-line work appears to be an important risk factor for anxiety, insomnia and overall psychological problems. Moreover, depression and symptoms of post-traumatic stress might persist even after such a crisis is over ${ }^{12} 1640$ and might exceed the consequences of the pandemic itself. ${ }^{19}$ These findings will help improve our understanding of the influence of pandemics on psychological health among healthcare workers and suggest implementation of steps that go beyond saving the lives of patients with COVID-19-psychosocial interventions and support should be integrated into public health responses to the COVID-19 pandemic, particularly for healthcare workers. Evidence-based psychosocial interventions and support for short-term psychological problems, such as anxiety, depression and insomnia, at the early stage of the pandemic are necessary. Moreover, self-relaxation training, regular exercise and healthy lifestyle should be emphasised. Additional studies should be conducted to explore the long-term impact of the COVID-19 pandemic (eg, depression and post-traumatic stress disorder) and psychosocial interventions to improve mental health.

Author affiliations

${ }^{1}$ Peking University Sixth Hospital, Peking University Institute of Mental Health, NHC Key Laboratory of Mental Health (Peking University), National Clinical Research Center for Mental Disorders (Peking University Sixth Hospital), Beijing, China ${ }^{2}$ National Institute on Drug Dependence and Beijing Key Laboratory on Drug Dependence Research, Peking University, Beijing, China

${ }^{3}$ Mental Health Institute of Inner Mongolia Autonomous Region, Hohhot, Inner Mongolia, China

${ }^{4}$ Xiamen City Xianyue Hospital, Xiamen, Fujian, China

${ }^{5}$ Peking-Tsinghua Center for Life Sciences and PKU-IDG/McGovern Institute for Brain Research, Beijing, China

${ }^{6}$ Department of Social Work and Social Administration, University of Hong Kong, Hong Kong, China

Acknowledgements We thank all the participants for their willingness to participate in the study and the time that they devoted to the study.

Contributors JQ and LL proposed the topic and the main idea. JQ analysed the data. JQ and SL wrote the initial draft. JD, JL, LZ, SW, YG, WH, KY, WY, YS, MR, YB and $L L$ commented on and revised the manuscript. LL made the final revision. All authors contributed to the final draft of the manuscript. 
Funding This study was supported by grants from the Beijing Natural Science Foundation (NO. 7194336), Special Research Fund of PKUHSC for Prevention and Control of COVID-19 (NO. BMU2020HKYZX008) and the National Natural Science Foundation of China (N0. 81761128036, 81821092 and 31900805).

\section{Competing interests None declared.}

\section{Patient consent for publication Not required.}

Ethics approval This study was approved by the ethical review committee of the Peking University Sixth Hospital.

Provenance and peer review Commissioned; externally peer reviewed.

Data availability statement Data are available upon reasonable request to the first author

Open access This is an open access article distributed in accordance with the Creative Commons Attribution Non Commercial (CC BY-NC 4.0) license, which permits others to distribute, remix, adapt, build upon this work non-commercially, and license their derivative works on different terms, provided the original work is properly cited, appropriate credit is given, any changes made indicated, and the use is non-commercial. See: http://creativecommons.org/licenses/by-nc/4.0/.

\section{ORCID ID}

Jianyu Que http://orcid.org/0000-0003-0660-5134

\section{REFERENCES}

1 World Health Organization. Coronavirus disease 2019 (COVID-19) Situation Report-82. Available: https://www.who.int/docs/defaultsource/coronaviruse/situation-reports/20200411-sitrep-82-covid-19. pdf?sfvrsn=74a5d15_2 [Accessed 15 Apr 2020].

2 Wang C, Horby PW, Hayden FG, et al. A novel coronavirus outbreak of global health concern. Lancet 2020;395:470-3.

3 Mahase E. Coronavirus: covid-19 has killed more people than SARS and MERS combined, despite lower case fatality rate. BMJ 2020;368:m641.

4 Baden LR, Rubin EJ. Covid-19 - The Search for Effective Therapy. N Engl J Med 2020;382:1851-2.

5 COVID-19: protecting health-care workers. Lancet 2020;395:922.

6 Catton H. Global challenges in health and health care for nurses and midwives everywhere. Int Nurs Rev 2020;67:4-6.

7 National Health Commission of the People's Republic of China. Available: http://www.nhc.gov.cn/xcs/fkdt/202003/a54a40ae2876 4f3581f36cc31204433c.shtml; [Accessed 28 Mar 2020].

8 Wang H, Wang S, Yu K. COVID-19 infection epidemic: the medical management strategies in Heilongjiang Province, China. Crit Care 2020;24:107.

9 Greenberg N, Docherty M, Gnanapragasam S, et al. Managing mental health challenges faced by healthcare workers during covid-19 pandemic. BMJ 2020;368:m1211.

10 Chen $\mathrm{Q}$, Liang M, Li Y, et al. Mental health care for medical staff in China during the COVID-19 outbreak. Lancet Psychiatry 2020;7:e15-16.

11 Kang L, Li Y, Hu S, et al. The mental health of medical workers in Wuhan, China dealing with the 2019 novel coronavirus. Lancet Psychiatry 2020;7:e14.

12 Chong MY, Wang WC, Hsieh WC, et al. Psychological impact of severe acute respiratory syndrome on health workers in a tertiary hospital. Br J Psychiatry 2004;185:127-33.

13 Maunder R. The experience of the 2003 SARS outbreak as a traumatic stress among frontline healthcare workers in Toronto: lessons learned. Philos Trans R Soc Lond B Biol Sci 2004;359:1117-25.

14 Maunder RG, Lancee WJ, Balderson KE, et al. Longterm psychological and occupational effects of providing hospital healthcare during SARS outbreak. Emerg Infect Dis 2006;12:1924-32

15 McAlonan GM, Lee AM, Cheung V, et al. Immediate and sustained psychological impact of an emerging infectious disease outbreak on health care workers. Can J Psychiatry 2007;52:241-7.

16 Wu P, Fang Y, Guan Z, et al. The psychological impact of the SARS epidemic on hospital employees in China: exposure, risk perception, and altruistic acceptance of risk. Can J Psychiatry 2009;54:302-11.

17 Lai J, Ma S, Wang Y, et al. Factors associated with mental health outcomes among health care workers exposed to coronavirus disease 2019. JAMA Netw Open 2020;3:e203976-e76.

18 Zhang WR, Wang K, Yin L, et al. Mental health and psychosocial problems of medical health workers during the COVID-19 epidemic in China. Psychother Psychosom 2020:1-9.
19 Bao Y, Sun Y, Meng S, et al. 2019-nCoV epidemic: address mental health care to empower society. Lancet 2020;395:e37-8.

20 World Health Organization. Global recommendations on physical activity for health. Available: https://www.who.int/dietphysicalactivity/ publications/9789241599979/en/; [Accessed 28 Mar 2020].

21 Department of Health. Alcohol guidelines review: report from the guidelines development group to the UK chief medical officers, 2016 Available: https://assets.publishing.service.gov.uk/government/ uploads/system/uploads/attachment_data/file/545739/GDG_reportJan2016.pdf; [Accessed 28 Mar 2020].

22 Li N, Wang H, Sun J, et al. An investigation on the mental health status of tobacco dependent soldiers. Chin J Behav Med Sci $2001 ; 10: 23$

$23 \mathrm{He}$ X, Li C, Qian J, et al. Reliability and validity of a generalized anxiety disorder scale in general hospital outpatients. Shanghai Arch Psychiatry 2010;22:200-3.

24 Spitzer RL, Kroenke K, Williams JBW, et al. A brief measure for assessing generalized anxiety disorder: the GAD-7. Arch Intern Med 2006;166:1092-7.

25 Löwe B, Decker O, Müller S, et al. Validation and standardization of the generalized anxiety disorder screener (GAD-7) in the general population. Med Care 2008;46:266-74.

26 Sun XY, Li YX, Yu CQ, et al. Reliability and validity of depression scales of Chinese version: a systematic review. Zhonghua Liu Xing Bing Xue Za Zhi 2017;38:110-6.

27 Spitzer RL, Kroenke K, Williams JB. Validation and utility of a selfreport version of PRIME-MD: the PHQ primary care study. primary care evaluation of mental disorders. patient health questionnaire. JAMA 1999;282:1737-44.

28 Kroenke K, Spitzer RL, Williams JB. The PHQ-9: validity of a brief depression severity measure. J Gen Intern Med 2001;16:606-13.

29 Li E, Li W, Xie Z, et al. Psychometric property of the insomnia severity index in students of a commercial school. J Neurosci Ment Health 2019;19:268-72.

30 Bastien $\mathrm{CH}$, Vallières $\mathrm{A}$, Morin $\mathrm{CM}$. Validation of the insomnia severity index as an outcome measure for insomnia research. Sleep Med 2001;2:297-307

31 Morin CM, Belleville G, Bélanger L, et al. The insomnia severity index: psychometric indicators to detect insomnia cases and evaluate treatment response. Sleep 2011;34:601-8.

32 Liu S, Yang L, Zhang C, et al. Online mental health services in China during the COVID-19 outbreak. Lancet Psychiatry 2020;7:e17-18.

33 Qiu J, Shen B, Zhao M, et al. A nationwide survey of psychological distress among Chinese people in the COVID-19 epidemic: implications and policy recommendations. Gen Psychiatry 2020;33:e100213.

34 Grace SL, Hershenfield K, Robertson E, et al. The occupational and psychosocial impact of SARS on academic physicians in three affected hospitals. Psychosomatics 2005;46:385-91.

35 Nickell LA, Crighton EJ, Tracy CS, et al. Psychosocial effects of SARS on hospital staff: survey of a large tertiary care institution. CMAJ 2004:170:793-8.

36 Maunder RG, Lancee WJ, Rourke S, et al. Factors associated with the psychological impact of severe acute respiratory syndrome on nurses and other hospital workers in Toronto. Psychosom Med 2004;66:938-42

37 Mo Y, Deng L, Zhang L, et al. Work stress among Chinese nurses to support Wuhan in fighting against COVID-19 epidemic. J Nurs Manag 2020. doi:10.1111/jonm.13014. [Epub ahead of print: $07 \mathrm{Apr}$ 2020].

38 Imai T, Takahashi K, Hasegawa N, et al. SARS risk perceptions in healthcare workers, Japan. Emerg Infect Dis 2005;11:404-10.

39 Phua DH, Tang HK, Tham KY. Coping responses of emergency physicians and nurses to the 2003 severe acute respiratory syndrome outbreak. Acad Emerg Med 2005;12:322-8.

40 Brooks SK, Dunn R, Amlôt R, et al. A systematic, thematic review of social and occupational factors associated with psychological outcomes in healthcare employees during an infectious disease outbreak. J Occup Environ Med 2018;60:248-57.

41 Pfefferbaum B, Newman E, Nelson SD, et al. Disaster media coverage and psychological outcomes: descriptive findings in the extant research. Curr Psychiatry Rep 2014;16:464

42 Hall BJ, Xiong YX, Yip PSY, et al. The association between disaster exposure and media use on post-traumatic stress disorder following typhoon Hato in Macao, China. Eur J Psychotraumatol 2019;10:1558709.

43 Harvey SB, Øverland S, Hatch SL, et al. Exercise and the prevention of depression: results of the HUNT cohort study. Am J Psychiatry 2018;175:28-36

44 Kessler RC, Bromet EJ. The epidemiology of depression across cultures. Annu Rev Public Health 2013;34:119-38. 
45 Chakravorty S, Chaudhary NS, Brower KJ. Alcohol dependence and its relationship with insomnia and other sleep disorders. Alcohol Clin Exp Res 2016;40:2271-82.

46 Roth T, Jaeger S, Jin R, et al. Sleep problems, comorbid mental disorders, and role functioning in the national comorbidity survey replication. Biol Psychiatry 2006;60:1364-71.

47 Tam CWC, Pang EPF, Lam LCW, et al. Severe acute respiratory syndrome (SARS) in Hong Kong in 2003: stress and psychological impact among frontline healthcare workers. Psychol Med 2004;34:1197-204.

48 Chua SE, Cheung V, Cheung C, et al. Psychological effects of the SARS outbreak in Hong Kong on high-risk health care workers. Can J Psychiatry 2004;49:391-3.

49 Wong WCW, Wong SYS, Lee A, et al. How to provide an effective primary health care in fighting against severe acute respiratory syndrome: the experiences of two cities. Am J Infect Control 2007;35:50-5.

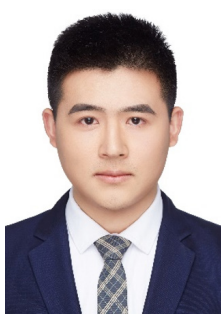

Jianyu Que is a PhD student in Peking University Sixth Hospital in Beijing, China. He obtained a bachelor's degree from Qiqihar Medical College in 2016 and got a master's degree from Peking University in 2019. His research interest includes mental health care of medical professionals. 